\title{
Polar mesosphere summer echoes: a comparison of simultaneous observations at three wavelengths
}

\author{
E. Belova, P. Dalin, and S. Kirkwood
}

Swedish Institute of Space Physics, Box 812, 98128 Kiruna, Sweden

Received: 25 April 2007 - Revised: 16 October 2007 - Accepted: 14 November 2007 - Published: 2 January 2008

\begin{abstract}
On 5 July 2005, simultaneous observations of Polar Mesosphere Summer Echoes (PMSE) were made using the EISCAT VHF $(224 \mathrm{MHz})$ and UHF $(933 \mathrm{MHz})$ radars located near Troms $\varnothing$, Norway and the ALWIN VHF radar (53.5 MHz) situated on Andøya, $120 \mathrm{~km} \mathrm{SW}$ of the EISCAT site. During the short interval from 12:20 UT until 12:26 UT strong echoes at about $84 \mathrm{~km}$ altitude were detected with all three radars. The radar volume reflectivities were found to be $4 \times 10^{-13} \mathrm{~m}^{-1}, 1.5 \times 10^{-14} \mathrm{~m}^{-1}$ and $1.5 \times 10^{-18} \mathrm{~m}^{-1}$ for the ALWIN, EISCAT-VHF and UHF radars, respectively. We have calculated the reflectivity ratios for each pair of radars and have compared them to ratios obtained from the turbulence-theory model proposed by Hill (1978a). We have tested different values of the turbulent energy dissipation rate $\varepsilon$ and Schmidt number $S_{c}$, which are free parameters in the model, to try to fit theoretical reflectivity ratios to the experimental ones. No single combination of the parameters $\varepsilon$ and $S_{c}$ could be found to give a good fit. Spectral widths for the EISCAT radars were estimated from the spectra computed from the autocorrelation functions obtained in the experiment. After correction for beam-width broadening, the spectral widths are about $4 \mathrm{~m} / \mathrm{s}$ for the EISCAT-VHF and $1.5-2 \mathrm{~m} / \mathrm{s}$ for the UHF radar. However, according to the turbulence theory, the spectral widths in $\mathrm{m} / \mathrm{s}$ should be the same for both radars. We also tested an incoherent scatter (IS) model developed by Cho et al. (1998), which takes into account the presence of charged aerosols/dust at the summer mesopause. It required very different sizes of particles for the EISCAT-VHF and UHF cases, to be able to fit the experimental spectra with model spectra. This implies that the IS model cannot explain PMSE spectra, at least not for monodisperse distributions of particles.
\end{abstract}

Keywords. Ionosphere (Ionosphere-atmosphere interactions) - Meteorology and atmospheric dynamics (Turbulence) - Electromagnetics (Scattering and diffraction)

Correspondence to: E. Belova

(belova@irf.se)

\section{Introduction}

Polar Mesosphere Summer Echoes (PMSE), strong radar returns from mesopause altitudes, have been the subject of intensive studies during the last 25 years (for reviews, see Cho and Röttger, 1997; Rapp and Lübken, 2004). It is widely believed in the research community that at least two conditions are necessary for PMSE occurrence: neutral turbulence and aerosol (ice) particles. Recently, one more polar mesospheric phenomenon, similar to PMSE but occurring in wintertime, has been re-examined, at first because of the extremely strong echoes observed during solar proton events. Weaker echoes have subsequently been observed often when D-region ionisation is enhanced above quiet levels. This phenomenon was named PMW(inter)E (Kirkwood et al., 2002; Kirkwood et al., 2006). Radar echoes like PMWE were detected earlier but on an irregular basis (probably because of a lack of regular observations of the polar mesosphere in wintertime) and were regarded as due to a neutral turbulence, without any critical analysis (e.g. Collis et al., 1992). More recently, PMWE were comprehensively studied and detected not only with MST radars at about $50 \mathrm{MHz}$ but also with the EISCAT VHF $(224 \mathrm{MHz})$ radar (Belova et al., 2005). Analysis of reflectivities and spectra within the PMWE altitude region (Kirkwood et al., 2006) has ruled out turbulence as a possible explanation of all aspects of the phenomenon. Highly damped ion-acoustic waves, resulting from the interaction of infrasound waves with atmospheric waves, have been proposed as a new, non-turbulent mechanism to explain PMWE features.

Belova et al. (2005) made use of radar backscatter at different frequencies, i.e. radiowave scattering from structures with different scale lengths, to study PMWE. Experimental values were compared to those expected on the basis of the theory of turbulence for passive scalar tracer (radar refractive index, in this case). However, for accurate calculation of theoretical reflectivities, one needs to assume values for some poorly-known constants related to normalization and to fitting of the model to the experiment (Hill, 1978a; Stebel

Published by Copernicus Publications on behalf of the European Geosciences Union. 
Table 1. Parameters of the radars.

\begin{tabular}{llll}
\hline & ALWIN & EISCAT VHF & EISCAT UHF \\
\hline Location & $69.2^{\circ} \mathrm{N}, 16^{\circ} \mathrm{E}$ & $69.6^{\circ} \mathrm{N}, 19.2^{\circ} \mathrm{E}$ & $69.6^{\circ} \mathrm{N}, 19.2^{\circ} \mathrm{E}$ \\
Frequency, $\mathrm{MHz}$ & 53.5 & 224 & 933 \\
Antenna 3-dB beam width & $6^{\circ}$ & $1.7^{\circ} \mathrm{NS} \times 1.2^{\circ} \mathrm{EW}$ & $0.5^{\circ}$ isotropically \\
Antenna area, m & & $2400(1 \mathrm{klystron})$ & 804 \\
Transmitter peak power, $\mathrm{kW}$ & 35 & $1500(1$ klystron $)$ & 1500 \\
\hline
\end{tabular}

et al., 2004). To avoid this uncertainty, it is possible instead to use the ratio of experimental reflectivities for two or more radars, working at different frequencies, for comparison with turbulence theory, in relation to PMWE or PMSE.

Röttger et al. (1990) were the first to report PMSE observed with 46.9-MHz and 933-MHz radars simultaneously. They calculated the reflectivity ratio for the radars, compared it with that expected from turbulence theory (for inertial range turbulence) and obtained a very large discrepancy between these two values. They explained this by an absence, at that time, of a proper theory to be applied for PMSE at $0.16 \mathrm{~m}$ length scale (corresponding to the Bragg wavelength for the 933-MHz radar). In the case of PMSE, the Schmidt number $S_{c}$ (the ratio of kinematic viscosity $v$ to plasma diffusivity D) might be high, due to the presence of charged aerosol particles and the turbulence spectrum can be extended to shorter length scales, beyond the inertial subrange. Further investigation has confirmed that $S_{c}$ during PMSE can be as high as 300-400 (Lübken et al., 1998; La Hoz et al., 2006) and for this case the Batchelor (1958) theory or the Hill (1978a) models may be applied for the turbulent spectrum of radar reflectivity at small scales.

Simultaneous observations of PMSE using the EISCAT VHF (224 MHz) and UHF (933 MHz) radars, located near Troms $\varnothing$, Norway were made during summer 2005 as part of an international PMSE-EISCAT campaign. The MST radar ALWIN (53.5 MHz), situated on Andøya, $120 \mathrm{~km} \mathrm{SW}$ of the EISCAT site, ran a special experiment during that time. During the short interval from 12:20 UT until 12:28 UT on 5 July, strong echoes at about $84 \mathrm{~km}$ were detected with all three radars. PMSE observation by UHF radar is a rather rare phenomenon. That was the first PMSE/EISCAT experiment where data were obtained from both radars simultaneously with a high enough quality to allow accurate spectral analysis. Here we aim to use these unique data to test the consistency with the existing turbulence-based theory and with incoherent scatter theory, taking into account the presence of charged particles at PMSE altitudes.

We start with a description of observations in the next section. We estimate radar reflectivities for all three radars, as well as spectral widths and Doppler shifts. Then, in Sect. 3, we give a short overview of the turbulence theory for a passive scalar tracer for high Schmidt numbers and obtain values to compare with the experiment in Sect. 4. Further, in Sect. 5, we discuss an incoherent scatter theory for multi-component plasma and apply it to the case of the measured PMSE. Finally, we discuss the results obtained.

\section{Observations}

We present data from the EISCAT VHF and UHF radars and from the ALWIN MST radar. The radar characteristics are described in Table 1. In July 2005, the EISCAT radars conducted special experiments for PMSE study. On 5 July both radars ran the experiment called "arc-dlayer_ht", which has a time resolution of $0.2 \mathrm{~s}$ and covers altitudes from $59.7 \mathrm{~km}$ to $139.5 \mathrm{~km}$, with a $300-\mathrm{m}$ range resolution. Beams were pointed vertically. Autocorrelation functions (ACFs) with 127 lags at 1.562-ms resolution were computed.

ALWIN was running a 216-s sequence of Doppler experiments with vertical and oblique beams ( $7^{\circ}$ off-zenith), combined with a spaced antenna experiment. The mesospheric observations have a time resolution of $51.2 \mathrm{~ms}$ and a range resolution of $300 \mathrm{~m}$. We use here vertical beam data obtained every $3.6 \mathrm{~min}$.

In Fig. 1 the observations from all three radars for the interval from 12:10 UT to 12:46 UT on 5 July 2005 are presented. The two lower panels show equivalent electron density as measured by the EISCAT radars and the upper panel shows a signal-to-noise ratio (SNR) for the ALWIN radar. We have calibrated the signal strength recorded by the EISCAT radars against the ionosonde (a Dynasonde) situated at the same site, in order to obtain correct values of the electron density at the peak of the E-region (see, e.g. Kirkwood et al., 1986). The EISCAT VHF and ALWIN radars show a strong and multi-layered PMSE between the $82-88-\mathrm{km}$ altitude during the entire time interval. Both radars show somewhat similar behaviour of the PMSE. One can notice that layers are double-peaked and descend slightly in the course of time, and that they are about the same thickness of $3-4 \mathrm{~km}$. Thus, we can assume that the properties of the agents causing the PMSE are the same or similar at these two locations. The UHF radar, on the other hand, detects a weak and single narrow layer of enhanced (equivalent) electron density at about $84 \mathrm{~km}$ from about 12:20 UT until 12:28 UT. The data from this last interval will be considered in more detail. 
We calculate maximum reflectivities for each radar, corresponding to altitudes of maximum backscattered power for the time interval of interest. For the EISCAT radars this is a straightforward procedure. The volume reflectivity $\eta$ is defined as $\sigma_{0} \cdot N_{e}$, where $\sigma_{0}=4.99 \times 10^{-29} \mathrm{~m}^{2}$ is the effective scatter cross section per electron and $N_{e}$ is the electron density (equivalent electron density for the case of PMSE) measured by the EISCAT radars. For calculation of ALWIN reflectivity, the radar calibration method described by Latteck et al. (2005) was used. The resulting radar reflectivities, averaged over the interval under consideration are: $\eta($ ALWIN $)=4 \times 10^{-13} \mathrm{~m}^{-1}$, $\eta(\mathrm{VHF})=1.5 \times 10^{-14} \mathrm{~m}^{-1}$ and $\eta(\mathrm{UHF})=1.5 \times 10^{-18} \mathrm{~m}^{-1}$. Combining the results for the different radars, we can calculate three ratios of radar reflectivities. Thus, $R_{e 1} \equiv \eta(\mathrm{ALWIN}) / \eta(\mathrm{VHF})=27 ; \quad R_{e 2} \equiv \eta(\mathrm{VHF}) / \eta(\mathrm{UHF})=10^{4}$ and $R_{e} \equiv \eta(\mathrm{ALWIN}) / \eta(\mathrm{UHF})=2.7 \times 10^{5}$. These experimental reflectivity ratios will be compared to those expected from turbulence theory in the next section.

The ACFs measured by the EISCAT radars can be Fouriertransformed to give PMSE power spectra. Two examples of the resulting power spectra (for 12:20 UT) are presented in Fig. 2. Left-hand panels show equivalent electron density profiles from EISCAT standard analysis. Power spectra for the VHF and UHF radars as a function of altitude averaged over $5 \mathrm{~min}$ and normalized by maximum spectral power are plotted on the right-hand panels. It is clearly seen that spectra can be resolved only where enhanced backscatter occurs and that they are rather narrow: about $20 \mathrm{~Hz}$ of spectral width for the UHF radar and less than $10 \mathrm{~Hz}$ for the VHF radar. We attempted to make longer integrations $(10 \mathrm{~min})$, in order to obtain spectra for other heights (e.g. over $90 \mathrm{~km}$ ) but without success. The reason for this may be that ordinary incoherent spectra in the E-region are of the order of $200-300 \mathrm{~Hz}$ wide for the VHF radar (see, e.g. Hall et al., 1987), which implies a few ms coherence time. The pulse-to-pulse correlation method used in this experiment is, in this case, unable to provide sufficient time resolution to resolve the spectra (other techniques are generally applied for E-region studies). In fact, for the "arc_dlayer" experiment there are 4 extra lags with $32 \mu$ s lag resolution available, but even that is not enough to resolve accurately the spectrum in the E-region. Inspection of Doppler shifts as a function of altitude shows that they do not vary too much, indicating that vertical winds do not vary significantly with height within the PMSE layer. Thus, gradients in the vertical winds do not contribute significantly to additional spectra broadening.

In order to obtain more accurate information about spectral width and Doppler shifts, we focus on spectra from the altitude where maximum backscattered power was observed. These are shown in Fig. 3 for the interval from about 12:21 UT to about 12:23 UT. The frequency offset from the origin, and the spectral width, may be interpreted as the mean of the line-of-sight velocities and their standard deviation in the scattering volume, respectively. Therefore, instead of fre-

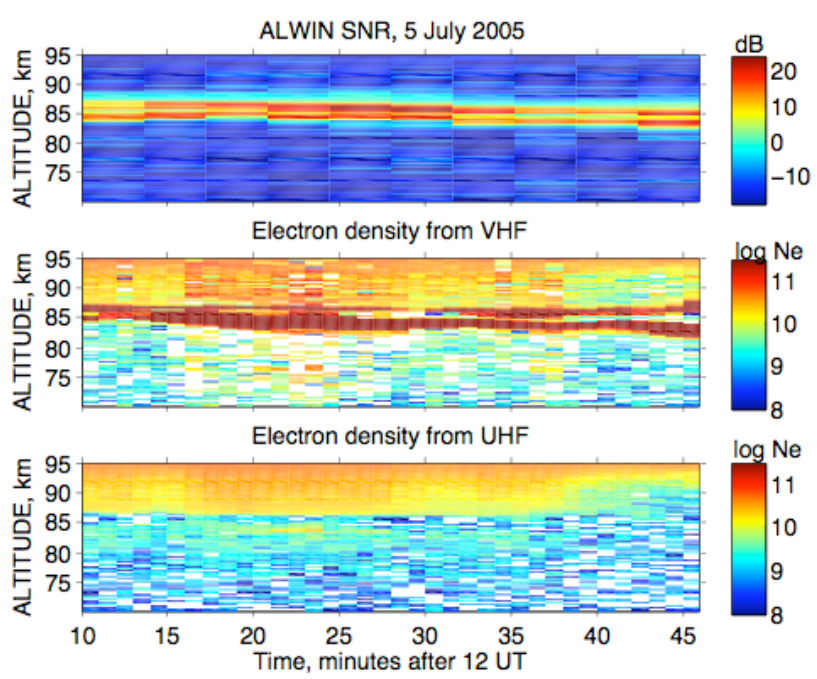

Fig. 1. Overview of radar observations on 5 July 2005 from 12:10 UT to 12:46 UT. Upper panel shows a radar echo signalto-noise ratio for ALWIN, the two lower panels show equivalent electron density derived from the EISCAT VHF (middle panel) and UHF (the lowest panel) radar measurements.

quency $\Omega$ we used the Doppler velocity $v_{D}$ as spectral axis, according to the formula: $v_{D}=c \cdot \Omega / 2 f_{r}$, where $c$ is a speed of light, $f_{r}$ is the radar frequency. The original data with 0.2 -s time resolution give very noisy spectra. We used postintegration over $6 \mathrm{~s}$ as a compromise between high noise level and spectral broadening due to long post-integration time. Spectra for the UHF radar have a higher noise level and often their shapes are far from ideal single Lorentzian or Gaussian functions (where the former is expected for incoherent spectra in the D-region of the ionosphere and the latter is expected for a coherent spectrum of turbulent origin). However, on some occasions, they are well enough defined to be able to be compared with those for VHF radar. We see from Fig. 3 that the VHF spectra are almost always wider than the UHF spectra. We chose two instances and compare them in more detail in Fig. 4. From this we see that the vertical bulk velocity defined by the mean Doppler shift is about $2 \mathrm{~m} / \mathrm{s}$ for both radars, but the spectral width is $1.5-2 \mathrm{~m} / \mathrm{s}$ for the UHF radar and $4 \mathrm{~m} / \mathrm{s}$ for the VHF radar.

\section{Turbulence theory for radar backscatter}

Neutral (air) turbulence is usually considered to be the main cause of irregularities in atmospheric parameters, and hence the cause of atmospheric radar echoes, at D-region heights. The parameters of turbulence can be directly determined from the spectrum of fluctuating velocity $u$ of the air, in wave number or length-scale space. Alternatively, measurements of a passive tracer such as, for example, temperature, electron density, or radar refractive index, can be used to 

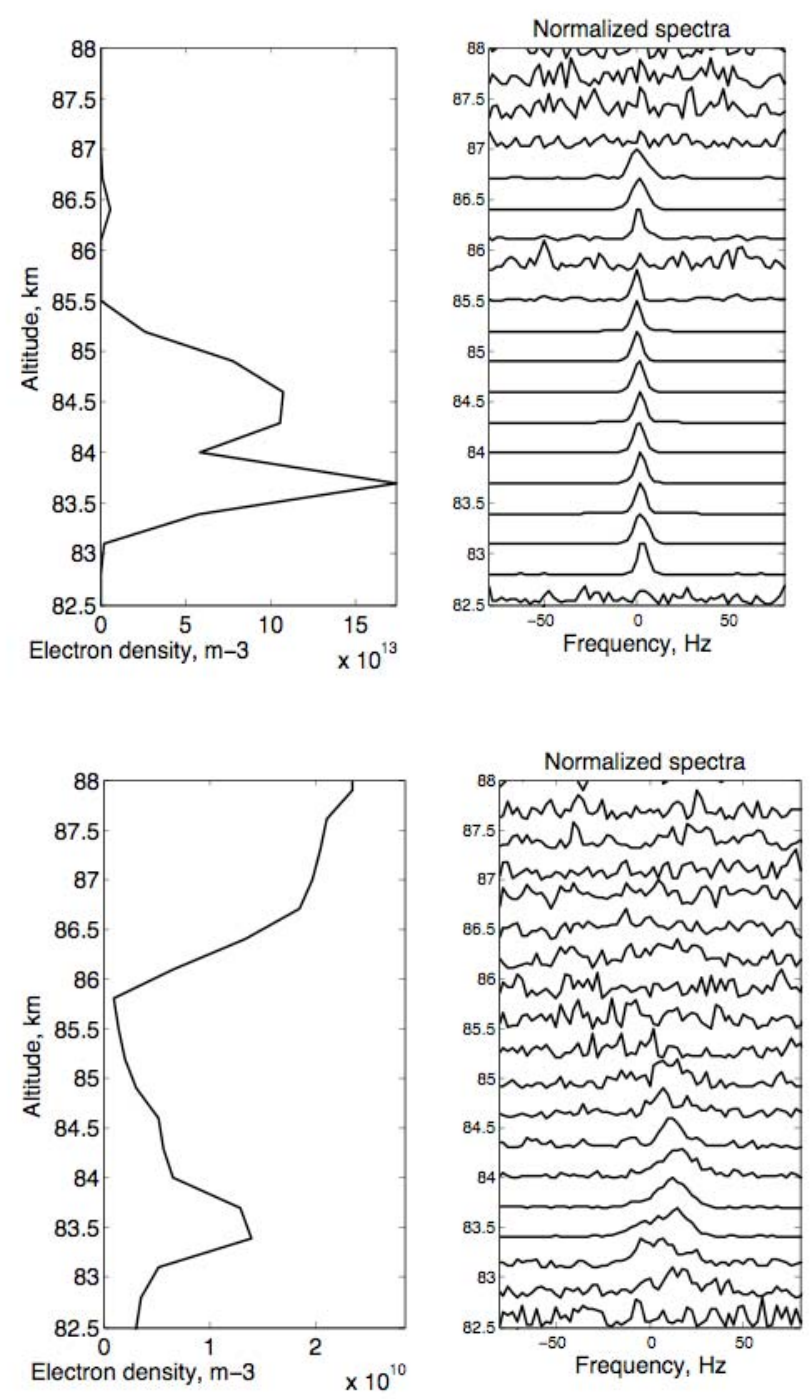

Fig. 2. Left-hand panels show the equivalent electron density profiles calculated from EISCAT VHF (upper panel) and UHF (lower panel) radar measurements at 12:20 UT. Right-hand panels present the spectra normalised by the echo power for VHF (upper panel) and UHF (lower panel) radars, averaged over 5 min.

derive turbulence parameters. Turbulent energy is primarily found at the largest length scales, from where it cascades to the smaller length scales and finally is dissipated as heat by diffusion at the smallest scales, or highest wave numbers. The part of the distribution of fluctuating velocity at large enough wave-numbers to be independent of viscosity is known as the inertial subrange, and the corresponding part in a passive scalar distribution, independent of molecular diffusion, is termed the convective subrange. Obuhov (1949) and Corrsin (1951) found that the spectrum $E$ of a passive scalar in the convective subrange follows Kolmogorov's law for the inertial subrange: $E(k) \sim k^{-5 / 3}$, for $k<<k_{0}$, where $k$ is a wave number and $k_{0}$ is a critical wave number corre- sponding to the Kolmogorov microscale $\lambda_{0}=\left(v^{3} / \varepsilon\right)^{1 / 4}$. Here $\varepsilon$ is the turbulent energy dissipation rate and $v$ is kinematic viscosity. For $k>k_{0}$, the spectrum $\mathrm{E}$ decays very rapidly. This theory was developed for $S_{c}=1$. Batchelor (1958) suggested that for $S_{c}>1$ the $-5 / 3$ power law is still valid for the inertial-convective subrange, where $k<<k_{0}$. Moreover, for $k \geq k_{0}$ he introduced viscous-convective and viscous-diffusive subranges with exponential $k^{-1} \cdot \exp \left(-D \cdot k^{2} / \gamma\right)$ law, where $\gamma=0.5 \cdot(\varepsilon / \nu)^{1 / 2}$. In the viscous-convective range, where $k_{0} \leq k<<k_{B}=k_{0} \cdot\left(S_{c}\right)^{1 / 2}$, the spectrum follows a $k^{-1}$ power law, producing a flattening in the spectral curve. Using these subranges, significant turbulent spectral power can extend to higher wave numbers than in the case when $S_{c}=1$. The theory for $S_{c}<1$ was developed by Batchelor et al. (1958), as well. However, no analytical form was proposed for the transition from inertial to viscous subranges. The gap was filled by Hill (1978a), who presented four models of the passive scalar spectrum for arbitrary values of $S_{c}$. The models have the same behaviour for the inertial-convective and viscousconvective subranges but differ in the diffusive ranges. The behaviour of the spectra in the viscous-diffusive subrange is the least certain part, due to the technical problems of measuring turbulence at such short length scales. For example, some scientists have suggested a more gentle decrease in this subrange compared to the Gaussian decrease predicted by Batchelor. All of Hill's models showed reasonable agreement with experiments in the different ranges. Driscoll and Kennedy (1985) expanded Hill's model 1 in a power series and made it universal in the sense of validity, not only for any $S_{c}$ values, but also for different Reynolds numbers. Despite its complexity (it requires numerical calculations of a power series), the Driscoll and Kennedy model has been widely used for deriving the turbulence dissipation rate and Schmidt numbers for PMSE conditions using the results of soundingrocket experiments (e.g. Lübken et al., 1994, 1998).

We want to apply the turbulence-spectrum theory for radar observations to our three radars described in Sect. 2. The passive tracer of turbulence in this case will be radar refractive index fluctuations at length-scales from $0.16 \mathrm{~m}$ for the EISCAT UHF radar to $2.8 \mathrm{~m}$ for the ALWIN radar, corresponding to wave numbers from $39 \mathrm{rad} / \mathrm{m}$ to $2.2 \mathrm{rad} / \mathrm{m}$, respectively. At the relevant altitudes the fluctuations in the refractive index are produced by those in the electron density. To decide which form of spectrum to apply to our observations, we have to define the subrange/subranges to which our radar data correspond. Lübken et al. (1998), on the basis of direct in-situ measurements of the scale-size spectrum of neutral density fluctuations in PMSE, found $\lambda_{0}=4.2 \mathrm{~m}$, and from this they derived $\varepsilon \approx 67 \mathrm{~mW} / \mathrm{kg}$. After fitting the model of Driscoll and Kennedy (1985) to the measured electron density fluctuation spectrum, they obtained a Schmidt number of about 400. This implies that the electron density fluctuations due to turbulence can lie beyond the inertial-convective subrange, even for the ALWIN radar. We will use one of Hill's models in the application to our observations of PMSE 

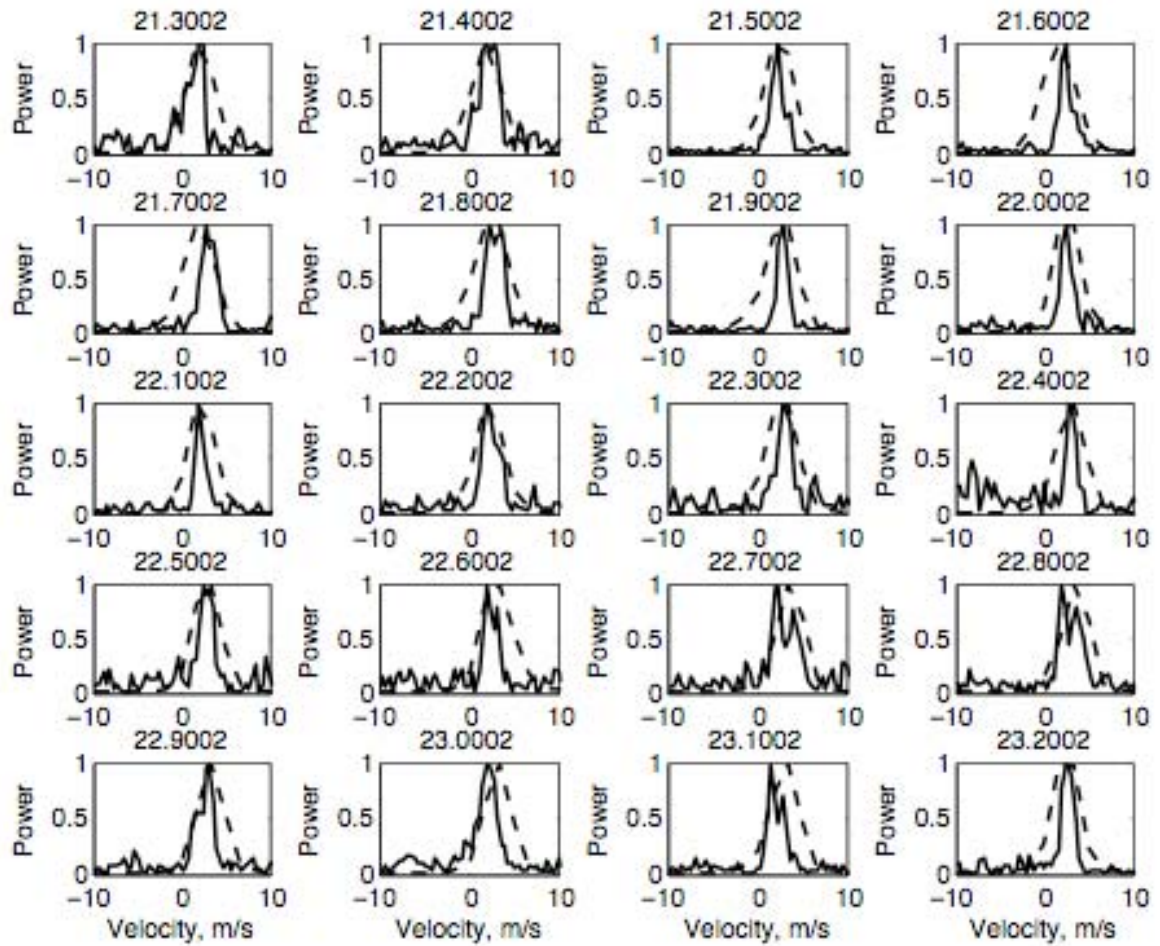

Fig. 3. Normalised spectra from the EISCAT UHF radar (solid line) and VHF radar (dashed line) calculated for the altitude of maximum backscattered power $(83.35 \mathrm{~km})$ for the time interval from 12:21:18 UT to 12:23:12 UT. $\mathrm{x}$-axis is in velocity units.

with three radars. For simplicity, we adopt Hill's model 1 in the following three-dimensional form, valid for radar experiments (which is obtained after multiplying one-dimensional spectrum by $k^{-2}$ ):

$$
\begin{aligned}
E_{N}(\tilde{k})= & A \cdot \chi \cdot \varepsilon^{-1 / 3} \cdot \lambda_{0}^{11 / 3} \cdot\left(\tilde{k}^{-11 / 3}+Q \tilde{k}^{-3}\right) . \\
& \exp \left[\frac{-\alpha}{S_{c}}\left(1.5 \tilde{k}^{4 / 3}+Q \tilde{k}^{2}\right)\right]
\end{aligned}
$$

where $E_{N}$ is the 3-D-spectrum of the electron density fluctuations, $\chi$ is their variance dissipation rate, $\tilde{k}=k \cdot \lambda_{0}$ is a non-dimensional wave number, and $A, Q$ and $\alpha$ are factors determined from comparison of experiments and numerical models.

Radar reflectivity $\eta$ for a radar with Bragg wave number $k$ can be expressed via the 3-D spectrum of electron density fluctuations $E_{N}(k)$ as (Tatarskii, 1961):

$\eta\left[m^{-1}\right]=B \cdot \bar{N}^{2} \cdot E_{N}(k)$,

where $B$ is a factor including several universal constants and $\bar{N}^{2}$ is the mean (true) electron density squared. Thus, absolute radar reflectivity is determined by four quantities, $\varepsilon, \chi$, $S_{c}$ and $N$, that are not measured directly. We use the ratio of the reflectivities, which allows us to decrease the number of parameters by two. We adopt $Q$ and $\alpha$ values of 2 and 0.83 , respectively, following Driscoll and Kennedy (1985), and use
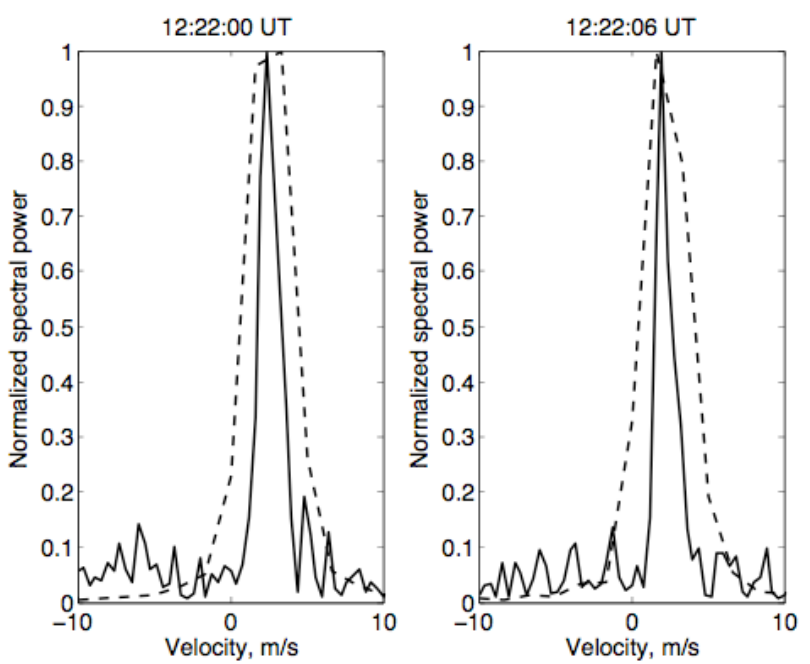

Fig. 4. Normalised spectra from the EISCAT UHF radar (solid line) and VHF radar (dashed line) calculated as for Fig. 3 but for two moments of time, as shown in the titles of panels.

a value of $\nu=0.95 \mathrm{~m}^{2} / \mathrm{s}$ which is representative for the $85-\mathrm{km}$ altitude, according to the MSIS90E model (Hedin, 1991).

Using Eqs. (1) and (2) we calculated radar reflectivity ratios combining any two radars. We 


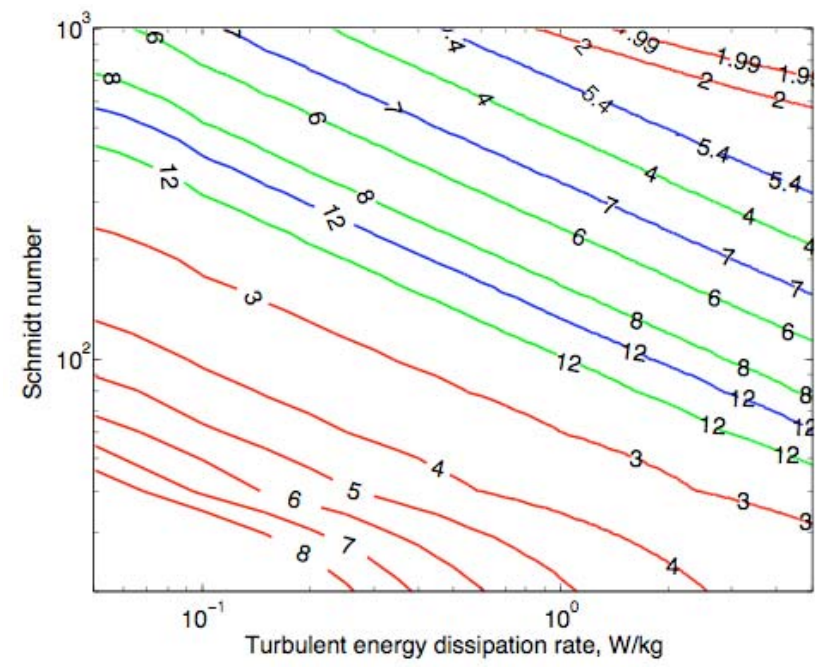

Fig. 5. Contours of $\log _{10}$ of reflectivity ratios calculated using turbulent theory for different turbulent energy dissipation rates and Schmidt numbers. Red lines are for the ALWIN/EISCAT VHF radar reflectivity ratio $R_{t 1}$, green lines are the EISCAT VHF/UHF radar reflectivity ratio $R_{t 2}$ and blue lines are the ALWIN/EISCAT UHF radar reflectivity ratio $R_{t 3}$. Logarithms of the experimental ratios are $\log _{10} R_{e 1}=1.4$ (out of graph); $\log _{10} R_{e 2}=4$ and $\log _{10} R_{e 3}=5.4$.

take $S_{c}=400$ and $\varepsilon=0.1 \mathrm{~W} / \mathrm{kg}$ as a somewhat moderate value for the $80-90 \mathrm{~km}$ altitude range during summertime, according to Lübken (1997). The results obtained are: $R_{t 1} \equiv E(\mathrm{ALWIN}) / E(\mathrm{VHF})=250$; $R_{t 2} \equiv E(\mathrm{VHF}) / E(\mathrm{UHF})=6.4 \times 10^{9} \quad$ and $\quad R_{t 3} \equiv E(\mathrm{ALWIN}) /$ $E(\mathrm{UHF})=1.6 \times 10^{12}$.

Let's now discuss what determines the spectral width for coherent radar signals. In general, it depends on the scatterers' velocity distribution in the illuminated volume. For the radars under discussion, the backscattered signal is from electron density fluctuations with a finite lifetime, so that spectral width also depends on the fluctuation lifetime. If the lifetime is long compared to the time used to calculate the spectrum, then spectra will be properly resolved and provide a measure of the velocity distribution of the scatters. On the other hand, if the lifetime is short compare to the time assigned in the radar experiment for computing the autocorrelation function (ACF), then the lifetime itself will determine the signal autocorrelation function and hence the spectral width. The lifetime of electron density fluctuations is the time required for ambipolar diffusion to destroy irregularities. For the PMSE case, electron diffusion is expected to be controlled by charged aerosol particles (Hill, 1978b), which makes the electron fluctuation lifetime very long. For example, for particles with a radius larger than $15 \mathrm{~nm}$, which is most likely during PMSE, the decay time of the fluctuations is estimated to be longer than tens of minutes (Rapp et al., 2003). Thus, the PMSE signal spectral width is a char- acteristic of the velocity distribution, and according to the turbulence theory, is proportional to the fluctuating turbulent velocity. The latter, in turn, is determined by the turbulence dissipation rate $\varepsilon$. The fluctuating velocity observed with a particular radar at a definite length-scale (Bragg scale) has an integrated effect on all scales in the turbulence,from the Bragg scale up to the largest scale (Hocking, 1985). However, as we described earlier, the amplitude of the velocity fluctuations is biggest for the larger length scales, thus the spectral width of a radar signal is determined by fluctuating velocity at these scales, regardless of the radar wavelength. Thus for pure turbulent scatter the spectral widths, expressed in $\mathrm{ms}^{-1}$, should be equal for all radars. In practice, spectral widths may differ due to beam- and shear spectral broadening.

\section{Discussion on turbulent reflectivities and spectra}

Röttger et al. (1990) estimated reflectivities for the CUPRI 46.9-MHz and the EISCAT UHF radars for a case of PMSE observations. They found for CUPRI $\eta=2 \times 10^{-12} \mathrm{~m}^{-1}$ and $\eta=1.2 \times 10^{-18} \mathrm{~m}^{-1}$ for the UHF radar. These values are close to those obtained by us in the present study (taking into account the frequency difference between ALWIN and CUPRI radars). Röttger et al. also estimated PMSE reflectivity for the EISCAT VHF radar on another occasion to be about $1.5 \times 10^{-16} \mathrm{~m}^{-1}$, which is 2 orders of magnitude less than we obtained. However, it turns out that the latter VHF radar reflectivity is probably too small to be typical, possibly due to very quiet geomagnetic conditions during that observation. Hocking and Röttger (1997) obtained a PMSE reflectivity for the EISCAT VHF radar of $1.3 \times 10^{-15} \mathrm{~m}^{-1}$. So the value $1.5 \times 10^{-14} \mathrm{~m}^{-1}$ for radar reflectivity which we obtained seems to be realistic for some conditions.

We can now compare the radar reflectivity ratios obtained from the experiment with those from turbulence theory. We see that experimental ratios are much less than the theoretical ones quoted above and the differences are larger when the highest radar frequencies (radar wave numbers) are considered. When calculating reflectivities from the theory in Sect. 3, we assumed the value of the energy dissipation rate $\varepsilon$ to be $0.1 \mathrm{~W} / \mathrm{kg}$ and the Schmidt number to be 400 . Within the PMSE layer, $\varepsilon$ has been estimated to vary from 1 to $1000 \mathrm{~mW} / \mathrm{kg}$ (Lübken et al., 2002) and the Schmidt number could vary, too. In Fig. 5 we plot the reflectivity ratios for all three radars as a function of $\varepsilon$ and $S_{c}$. Values of theoretical ratios that are close to the observed ones lie in the upper right corner of the figure, i.e. where $\varepsilon$ and $S_{c}$ are high enough. If turbulence theory fits ideally to the experiment, then the contours for the three reflectivity ratios with values equal to the experimental ones should cross at some point, which would determine one set of $\varepsilon$ and $S_{c}$. However, the contours with values of $R_{t 2}=10^{4}$ and $R_{t 3}=10^{5.4}$ (equal to the experimental values) are parallel and some distance from each other. The 
Schmidt numbers and turbulent dissipation rates for these contours differ by about 1.5 times and 2.3 times, respectively. As has already been mentioned, the theory is most uncertain for larger wave numbers, within the viscous-diffusive subrange, i.e. for $\lambda<<\lambda_{0} \cdot\left(S_{c}\right)^{-1 / 2} \approx 0.21 \mathrm{~m}$. Thus, one can expect that the EISCAT UHF radar lies within this subrange, which might result in large uncertainties in the estimation of the $R_{t 2}$ and $R_{t 3}$ values. However, it turns that $R_{t 1}$, which should be estimated most reliably, is about 4 times larger than $R_{e 1}$, even for extremely high energy dissipation rates, such as $5 \mathrm{~W} / \mathrm{kg}$ and $S_{c}=1000$. In order for theoretical reflectivity ratios to match the experimental, unrealistically high values of $S_{c}$ are needed. Thus, we cannot find one set of $\varepsilon$ and $S_{c}$ within reasonable limits, which would give us theoretical reflectivity ratios close to those obtained from the observations.

We see at least two possibilities to explain our results concerning radar reflectivities. The first is that the existing theories of turbulence for high the Schmidt number, which have been widely used in numerous PMSE studies, are not correct for the viscous-convective and viscous-diffusive subranges. More work is needed in this direction. Another possible explanation is that parameters of turbulence were very different in the ALWIN and EISCAT radar scattering volumes. Three collocated radars are needed to check our results. This may be possible in the future when a new MST radar will be installed near EISCAT (Ove Havnes, Tromsø University, personal communication). (If the present results are confirmed, this may be an argument against the hypothesis of a turbulent origin of PMSE.) However, there is one significant factor which does not allow us to draw a convincing conclusion about the turbulent origin of echoes on the bases of radar reflectivities only. That is the "filling factor" $(F)$, which is the fraction of the scattering volume, which is filled by turbulence. When calculating experimental radar reflectivities, $F$ has been assumed to be 1 or at least the same for each radar. This assumption cannot be proved because the scattering volumes are very different, not only between the ALWIN and EISCAT radars located at different places, but also for the collocated VHF and UHF radars which have different radiated beams (see Table 1). Therefore, we have to also consider radar backscatter spectral widths.

When considering the radar backscatter spectral widths for different radars, care must be taken to accurately separate the turbulent part from the nonturbulent contribution (Hocking, 1983). For a vertical beam the latter is primarily due to beam-width spectral broadening. Antenna beam widths for the 3 radars can be found in Table 1. For the EISCAT UHF radar the antenna beams width of $0.5^{\circ}$ (circular beam) is rather small and spectral broadening can be neglected. However, strong horizontal winds could contribute to (nonturbulent) spectral broadening for the other two radars, mainly for the ALWIN radar, but also for the EISCAT-VHF radar. Here we use only the EISCAT radars because the beamwidth broadening for ALWIN would make for much higher

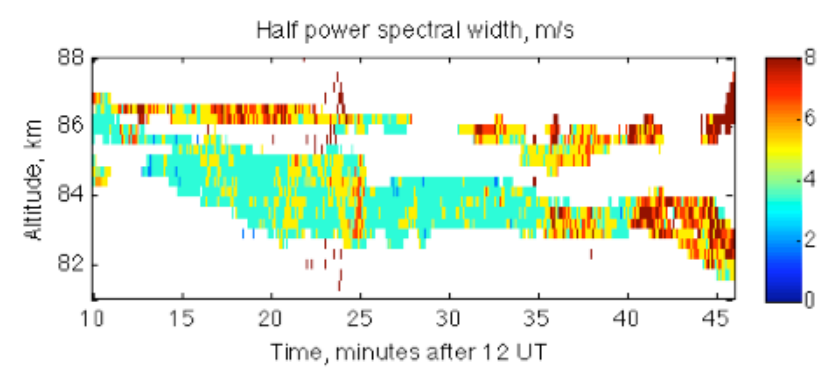

Fig. 6. Half-power spectral width of PMSE on 5 July 2004, calculated from the EISCAT VHF radar spectra with 6-s integration. Colorbar shows spectral width scale in $\mathrm{m} / \mathrm{s}$.

uncertainty. We have obtained estimates for the horizontal winds from the measurements over Esrange $\left(68^{\circ} \mathrm{N}, 21^{\circ} \mathrm{E}\right)$ by the SkiYMET meteor radar operated by University of Bath, UK and have then calculated the turbulent spectral widths for the radars using a procedure described by Hocking (1985). First, we calculate the "nonturbulent spectral width" $\Delta w_{\text {nont }}$, due to spectral broadening, for the different beamwidths of the VHF and UHF radars (Atlas, 1964; Hocking, 1985): $\Delta w_{\text {nont }}(\mathrm{m} / \mathrm{s}) \approx 0.6 \cdot \theta \cdot V_{\text {hor }}$, where $V_{\text {hor }} \approx 37 \mathrm{~m} / \mathrm{s}$ is the horizontal wind derived from SkiYMET measurements, for the $82-86 \mathrm{~km}$ altitude range, between 12:00 UT and 12:30 UT, and $\theta$ is the half-power full-width for the transmission (or reception) polar diagram in the direction of $V_{\text {hor }}$. Because the zonal component of $V_{\text {hor }}$ is more than 5 times larger than the meridional component, we can neglect the latter. Then $\theta=1.2^{\circ}, \Delta w_{\text {nont }}(\mathrm{VHF}) \approx 0.5 \mathrm{~m} / \mathrm{s}$, for the VHF radar and $\theta=0.5^{\circ}, \Delta w_{\text {nont }}(\mathrm{UHF})=0.2 \mathrm{~m} / \mathrm{s}$ for the UHF radar. Turbulent spectral widths are computed according to the formula $\left(\Delta w_{\text {tur }}\right)^{2} \approx\left(\Delta w_{\text {obs }}\right)^{2}-\left(\Delta w_{\text {nont }}\right)^{2}$. Finally, we obtain $\Delta w_{\text {tur }}(\mathrm{VHF})=3.97 \mathrm{~m} / \mathrm{s}$ and $\Delta w_{\text {tur }}(\mathrm{UHF})=1.99 \mathrm{~m} / \mathrm{s}$. Thus, the PMSE spectra for the VHF radar are almost twice as broad as those for the UHF radar, which is in contradiction to the concept that they are determined purely by turbulence.

La Hoz et al. (2006) have shown that the spectral width of the PMSE signal measured with the EISCAT UHF can be rather variable. It changed from $4 \mathrm{~m} / \mathrm{s}$ to $16 \mathrm{~m} / \mathrm{s}$ within $20 \mathrm{~min}$. During the $7 \mathrm{~min}$ of our PMSE UHF observations, the spectral widths of the echoes do not show significant changes. In order to see how typical the values are that we obtained for the VHF radar, we have calculated and plotted in Fig. 6 the PMSE spectral widths for this radar for the longer period from 12:10 to 12:46 UT. We see that for the strongest lower layer, the spectral width is about $4 \mathrm{~m} / \mathrm{s}$ most of the time, but can reach up to about $7 \mathrm{~m} / \mathrm{s}$. UHF PMSE occurred at altitudes close to the maximum of this layer. For the upper layer the spectra are generally broader than for the lower layer. However, during the entire interval the spectral width is never as narrow as the $2 \mathrm{~m} / \mathrm{s}$ obtained for the UHF radar. Additionally, at altitudes and times when the strongest PMSE were observed (from 12:15 UT to 12:28 UT, see Fig. 1, the 
middle panel) the spectra are no broader, as might be expected if some nonlinearity in the receiver was artificially broadening the spectra.

Hocking (1985) has suggested that, for scatters well within dissipation subrange, the eddies with scales in this region could be intermittent and decouple from the larger eddies. Then the fluctuating velocity measured by the radar would be due only to contributions from eddies with the radar Bragg scale, or close to it, rather than the integrated effect of the fluctuating motions at all length scales. In this case spectral widths might be different for radars of different frequencies. The eddies at the Bragg-scale for the EISCAT UHF radar can lie deeply in the viscous-diffusive subrange, if $S_{c}$ and $\varepsilon$ are not big enough; however, then we would not expect to observe PMSE with the UHF radar at all.

\section{Incoherent scatter spectra for complex dusty plasma}

Normal incoherent scatter echoes measured by the EISCAT radars are due to thermal fluctuations in electron density. The summer mesopause, a region in the neutral atmosphere between 80 and $90 \mathrm{~km}$, where the neutral temperature reaches its global minimum and ice particles are formed, corresponds to the D-region in the ionized part of the atmosphere - the ionosphere. Here fluctuations in electron density are affected by those in other ionized constituents such as ions and charged particles. The standard procedure used for deriving incoherent scatter spectra for EISCAT takes into account only the contribution of ions and hence cannot be applicable for the case of PMSE. We use the model by Cho et al. (1998), based on the fluid-equation approach originally proposed by Tanenbaum (1968), for the incoherent scatter spectrum for the complex dusty plasma observed in the PMSE region in the following form:

$$
\begin{array}{r}
S\left(\omega_{0}+\omega\right) d \omega=\frac{r_{e}^{2} N_{e} d \omega}{\pi \omega}\left|\frac{1}{\alpha_{e}^{2}+z_{e}\left[1+\sum_{s \neq e} \frac{\alpha_{s}^{2}}{z_{s}}\right]}\right|^{2} \\
\operatorname{Im}\left(z_{e}\left|1+\sum_{s \neq e} \frac{\alpha_{s}^{2}}{z_{s}}\right|^{2}+\frac{\alpha_{e}^{2}}{T_{e}} \sum_{s \neq e} T_{s} \frac{\alpha_{s}^{2}}{z_{s} *}\right),
\end{array}
$$

where $\omega$ is the Doppler frequency shift from the radar frequency $\omega_{0}, r_{e}$ - the classical electron radius, $N_{e}$ - the electron number density, $\alpha_{e}$ and $\alpha_{s}$ - the radar Bragg length normalized by the Debye lengths of electron and plasma constituents, respectively, $z_{s}\left(z_{e}\right)$ - functions of the constituent (electron)-neutral collision frequency and mass, and the mean thermal velocity of plasma constituents, $T_{e}$ and $T_{s}$ - temperatures of electron and plasma constituents. The asterisk denotes the complex conjugate.

The electron-neutral collision frequency and non-electron constituent collision frequency are calculated by Banks and Kockarts (1973) and by Schunk (1975). Temperatures of electron and plasma constituents are considered to be equal to the neutral temperature of the atmosphere. The neutral temperature, as well as neutral number density of the atmosphere, are obtained from the MSISE90 model (http: //modelweb.gsfc.nasa.gov/models/msis.html).

In the present modelling we assume that plasma in the Dregion is composed of the following constituents: free electrons, positive ions and positively charged meteoric dust or ice particles. We choose positively charged particles because the introduction of negatively charged particles into the model leads to unrealistic model spectra, which do not match the experimental ones under the expected prevailing conditions. The negative ion number density under summer mesosphere conditions is expected to be negligibly small (Kirkwood and Osepian, 1995). The model is self-consistent in the sense that the positive ion number density is computed in such a way as to preserve the quasi-neutrality of the multicomponent plasma. The mean mass of the positive ions is 31 A.M.U., corresponding to a mixture of $\mathrm{NO}^{+}$and $\mathrm{O}_{2}^{+}$. We do not take into account cluster ions (their possible influence will be considred in future work). The mass density for the charged dust component of the plasma is assumed to be $2 \mathrm{~g} / \mathrm{cm}^{3}$ (Hunten et al., 1980). As an input parameter for the model (applied for the altitude of maximum backscatter for the 80-90 km altitude range), we use the electron density at this height. This has been calculated using the electron density above and below the PMSE layer (as given by standard EISCAT data analysis) and then interpolating over the PMSE region. We assume that aerosol particles have a single positive charge (Rapp and Lübken, 1999), which, according to Cho et al. (1998), is a good assumption for aerosols of up to about a 10-nm radius. We compute the dust particle number density and size to give the best fit of the model to the experimental spectra. In Fig. 7 the modelled and experimental spectra are shown for the EISCAT VHF and UHF radar at 12:23 UT. Single positively charged particles with number density of $2.7 \times 10^{9} \mathrm{~m}^{-3}$ and a $2.1-\mathrm{nm}$ radius provide the best fit to the experimental spectrum for the UHF radar; however, particles with the same density but with a $0.8-\mathrm{nm}$ radius are the best for the VHF radar, i.e. charged dust particles of the same size have an impact in different ways on the amplitude and shape of the VHF and UHF incoherent scatter spectra.

\section{Conclusions}

We have analysed PMSE spectra and radar reflectivities at three Bragg scales: $3 \mathrm{~m}, 0.67 \mathrm{~m}$ and $0.16 \mathrm{~m}$, that is for the ALWIN, EISCAT VHF and EISCAT UHF radars, respectively. We have calculated reflectivity ratios for each pair of radars and compared to ratios obtained from the turbulencetheory model appropriate to our radars' wave numbers. We cannot find any set of values for turbulent energy dissipation rate $\varepsilon$ and Schmidt number $S_{c}$ for which the theoretical reflectivity ratios are close to those obtained from the 

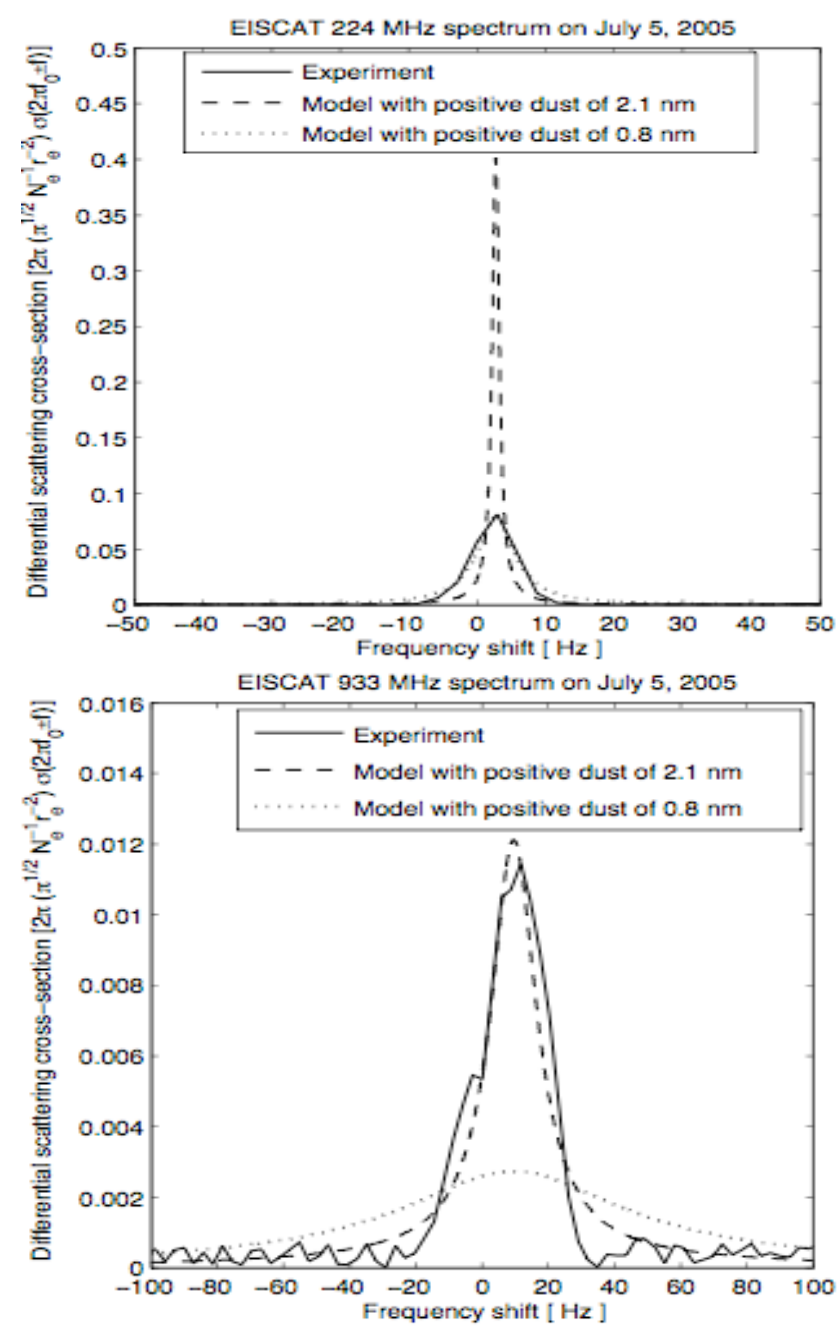

Fig. 7. Normalized spectra for EISCAT VHF (upper panel) and UHF (lower panel) radars. Solid curves are for experimental data at 12:23 UT, averaged over $1 \mathrm{~min}$. Dashed curves are model spectra (Cho et al., 1998), assuming singly positively charged particles with a 2.1-nm radius and dotted curves are those with a $0.8-\mathrm{nm}$ radius. For both cases: the electron number density is $3000 \mathrm{~cm}^{-3}$, concentration of singly positively charged particles is $2700 \mathrm{~cm}^{-3}$, concentration of positive ions is $300 \mathrm{~cm}^{-3}$. The ratio of negative ions to electron number densities is $4 \times 10^{-5}$.

experiment. This could be due to inadequacies in the turbulence theory for the high wave numbers and high Schmidt numbers relevant to our experimental data. Another possibility is that the parameters of the turbulence causing PMSE were very different in the two observation volumes.

Spectral widths for the EISCAT VHF radar (in $\mathrm{ms}^{-1}$ ) are found to be twice those for the UHF radar, in contradiction to what is expected from turbulence theory.

An incoherent scatter (IS) model of spectral shape requires very different sizes of particles to explain the spectra measured by the VHF and UHF radars, respectively, when the models are adjusted to fit the experimental spectra as closely as possible. This implies that the IS model cannot explain the PMSE spectra, at least not for a mono-disperse distribution of particles.

Acknowledgements. The authors thank R. Latteck and W. Singer for providing the ALWIN data, N. J. Mitchell and P. T. Younger for the horizontal wind estimates from the SkiYMET radar and M. Rietveld for the data on EISCAT radar calibrations against the Dynasonde. We acknowledge also J. Ekeberg who participated in the EISCAT-PMSE experiment in July 2005. The EISCAT Scientific Association is supported by the research councils of China, Finland, France, Germany, Japan, Norway, Sweden and UK.

Topical Editor U.-P. Hoppe thanks C. Hall and another anonymous referee for their help in evaluating this paper.

\section{References}

Atlas, D.: Advances in radar meteorology, Adv. Geophys., 10, 317479, Academic Press, New York, 1964.

Banks, P. M. and Kockarts, G.: Aeronomy, Part A., Academic Press, New York, 1973.

Batchelor, G. K.: Small-scale variation of convected quantities like temperature in turbulent fluid, Part 1. General discussion and the case of small conductivity, J. Fluid Mech., 5, 113-133, 1958.

Batchelor, G. K., Horwells, I. D., and Townsend, A. A.: Smallscale variation of convected quantities like temperature in turbulent fluid, Part 2. The case of large conductivity, J. Fluid Mech., 5, 134-139, 1958.

Belova, E., Kirkwood, S., Ekeberg, J., Osepian, A., Haggström, I., Nilsson, H., and Rietveld, M.: The dynamic background of Polar Mesosphere Winter Echoes from simultaneous EISCAT and ESRAD observation, Ann. Geophys., 23, 1239-1247, 2005, http://www.ann-geophys.net/23/1239/2005/.

Cho, J. Y. N. and Röttger, J.: An updated review of polar mesosphere summer echoes: Observation, theory and their relationship to noctilucent clouds and subvisible aerosols, J. Geophys. Res., 102, 2001-2020, 1997.

Cho, J. Y. N., Sulzer, M. P., and Kelley, M. C.: Meteoric dust effects on D-region incoherent scatter spectra, J. Atmos. Sol.-Terr. Phy., 60, 349-357, 1998.

Collis, P. N., Rietveld, M. T., Röttger, J., and Hocking, W. K.: Turbulence scattering layers in the middle-mesosphere observed by the EISCAT $224 \mathrm{MHz}$ radar, Radio Sci., 27, 97-107, 1992.

Corrsin, S.: On the spectrum of isotropic temperature fluctuations in isotropic turbulence, J. Appl. Phys., 22, 469-473, 1951.

Driscoll, R. J. and Kennedy, L. A.: A model for the spectrum of passive scalars in an isotropic turbulence field, Phys. Fluids, 28, 72-80, 1985.

Hall, C. M., Hoppe, U.-P., Williams, P. J. S., and Jones, G. O. L.: Mesospheric measurements using the EISCAT VHF system: first results and their interpretation, Geophys. Res. Lett., 14, 11871190, 1987.

Hedin, A. E.: Extension of the MSIS Thermospheric Model into the Middle and Lower Atmosphere, J. Geophys. Res., 96, 1159$1172,1991$.

Hill, R. J.: Models of the scalar spectrum for turbulent advection, J. Fluid Mech., 88, 541-562, 1978a. 
Hill, R. J.: Nonneutral and quasi-neutral diffusion of weakly ionized multiconstituent plasma, J. Geophys. Res., 83, 989-998, 1978b.

Hocking, W. K.: On the extraction of atmospheric turbulence parameters from radar backscatter Doppler spectra - I. Theory, J. Atmos. Sol.-Terr. Phy., 45, 89-102, 1983.

Hocking, W. K.: Measurement of turbulent energy dissipation rates in the middle atmosphere by radar techniques, Radio Sci., 20, 1403-1422, 1985.

Hocking, W. K. and Röttger, J.: Studies of polar mesosphere summer echoes over EISCAT using calibrated signal strengths and statistical parameters, Radio Sci., 32, 1425-1444, 1997.

Hunten, D. M., Turco, R. P., and Toon, O. B.: Smoke and dust particles of meteoric origin, in the mesosphere and stratosphere, J. Atmos. Sci., 37, 1342-1357, 1980.

Kirkwood, S., Collis P. N., and Schmidt, W.: Calibration of electron densities for the EISCAT UHF radar, J. Atmos. Terr. Phy., 48, 773-775, 1986.

Kirkwood, S. and Osepian, A.: Quantitative studies of energetic particle precipitation using incoherent scatter radar, J. Geomag. Geoelectr., 47, 783-799, 1995.

Kirkwood, S., Barabash, V., Belova, E., Nilsson, H., Rao, N., Stebel, K., Osepian, A., and Chilson, P. B.: Polar mesosphere winter echoes during solar proton events, Advances in Polar Upper Atmosphere Research, 16, 111-125, 2002.

Kirkwood, S., Chilson, P., Belova, E., Dalin, P., Häggström, I., Rietveld, M., and Singer, W.: Infrasound - the cause of strong Polar Mesosphere Winter Echoes?, Ann. Geophys., 24, 475-491, 2006, http://www.ann-geophys.net/24/475/2006/.

La Hoz, C., Havnes, O., Naeshem, L. I., and Hysell, D. L.: Observations and theories of Polar Mesospheric Summer Echoes at a Bragg wavelength of $16 \mathrm{~cm}$, J. Geophys. Res., 111, D04203, doi:10.1029/2005JD006044, 2006.

Latteck, R., Singer, W., Kirkwood, S., Jönsson, L. O., and Eriksson, H.: Observation of mesosphere summer echoes with calibrated VHF radars at latitudes between $54^{\circ} \mathrm{N}$ and $69^{\circ} \mathrm{N}$ in summer 2004, in Proceedings of the 17th ESA Symposium on European Rocket and Balloon Programmes and Related Research, Sandefjord, Norway (ESA SP-590), 29.5.-2.6.2005, 2005.

Lübken, F.-J., Giebeler, J., Blix, T., Thrane, E., Singer, W., and Bremer, J.: In-situ measurement of the Schmidt number within a PMSE layer, Geophys. Res. Lett., 21, 1651-1654, 1994.
Lübken, F.-J., Rapp, M., Blix, T., and Thrane, E.: Microphysical and turbulent measurements of the Schmidt number in the vicinity of polar mesosphere summer echoes, Geophys. Res. Lett., 25, 893-896, 1998.

Lübken, F.-J.: Seasonal variation of turbulent energy dissipation rates at high latitudes as determined by in situ measurements of neutral density fluctuations, J. Geophys. Res., 102, $13441-$ $13456,1997$.

Lübken, F.-J., Rapp, M., and Hoffmann, P.: Neutral air turbulence and temperatures in the vicinity of polar mesosphere summer echoes, J. Geophys. Res., 107(D15), 4273, doi:10.1029/2001JD000915, 2002.

Obuhov, A. M.: Structure of the temperature field in turbulent flow, Izv. Akad. Nauk SSSR, Ser. Geogr. I Geofiz., 13, 58-69, 1949.

Rapp, M. and Lübken, F.-J.: Modelling of positively charged aerosols in the polar summer mesopause region, Earth Planets Space, 51, 799-807, 1999.

Rapp, M., Lübken, F.-J., and Blix, T. A.: The role of charged ice particles for the creation of PMSE: a review of recent developments, Adv. Space Res., 31(9), 2033-2043, 2003.

Rapp, M. and Lübken, F.-J.: Polar mesosphere summer echoes (PMSE): review of observations and current understanding, Atmos. Chem. Phys., 4, 2601-2633, 2004, http://www.atmos-chem-phys.net/4/2601/2004/.

Röttger J., Rietveld, M. T., La Hoz, C., Hall, T., Kelley, C., and Swartz, W. E.: Polar mesosphere summer echoes observed with the EISCAT $933 \mathrm{MHz}$ radar and the CUPRI $46.9 \mathrm{MHz}$ radar, their similarity to $224 \mathrm{MHz}$ radar echoes and their relation to turbulence and electron density profiles, Radio Sci., 25, 671-687, 1990.

Schunk, R. W.: Transport equations for aeronomy, Planet. Space Sci., 23, 437-485, 1975.

Stebel, K., Blum, U., Fricke, K.-H., Kirkwood, S., Mitchell, N., and Osepian, A.: Joint radar/lidar observations of possible aerosol layers in the winter mesosphere, J. Atmos. Sol.-Terr. Phy., 66, 957-970, 2004.

Tanenbaum, B. S.: Continuum theory of Thomson scattering, Phys. Rev., 171, 215-221, 1968.

Tatarskii, V. I.: Wave propagation in a turbulent medium, McGrawHill, New York, 1961. 